may be employed at any given time. This is one of the characteristics of teaching behavior that creates difficulties for investigators, but makes the behavior so endlessly interesting.

\section{Measuring teaching through hormones and time series analysis: Towards a comparative framework $^{1}$}

\section{doi:10.1017/S0140525X14000806, e58}

\section{Andrea Ravignani and Ruth Sonnweber \\ Department of Cognitive Biology, University of Vienna, A-1090 Vienna, Austria. andrea.ravignani@gmail.com ruth-sophie.sonnweber@univie.ac.at http://homepage.univie.ac.at/andrea.ravignani https://www.researchgate.net/profile/Ruth_Sonnweber}

\begin{abstract}
Arguments about the nature of teaching have depended principally on naturalistic observation and some experimental work. Additional measurement tools, and physiological variations and manipulations can provide insights on the intrinsic structure and state of the participants better than verbal descriptions alone: namely, timeseries analysis, and examination of the role of hormones and neuromodulators on the behaviors of teacher and pupil.
\end{abstract}

We welcome Kline's emphasis on comparing behavioral measurements between learning episodes, and suggest including novel measures applicable to taught/learnt behaviors across species and tasks.

Hormonal or neuromodulatory states are well known to affect learning, for example, in bird-song acquisition (Ball et al. 2002). So far, little research has been undertaken on the role of hormonal states of teachers and learners in teaching episodes, although such examination offers promise for intraspecies and interspecies comparisons. The few animal studies linking endocrine parameters with social learning show that oxytocin and arginine-vasopressin mediate the social transmission of food preferences and that oxytocin plays an important role in mate-choice copying (Dore et al. 2013). Until more direct evidence is available, predictions on the endocrinology of teaching can be established by linking hormonal measures to behaviors that are essential to teaching. For instance, oxytocin and vasopressin mediate social approach and aversion (Porges 2001). Arguably, increased approach motivation and decreased social aversion are essential in teaching contexts. Other relevant behaviors, such as social motivation, affiliation, individual recognition, aggression, anxiety, and stress are associated with and regulated by oxytocin, vasopressin, testosterone, estrogens, and progesterone (McCall \& Singer 2012; Mehta \& Josephs 2012). These hormones also regulate and are influenced by trust, prosociality, empathy (empathic concern, perspective taking), reward sensitivity, and status seeking (Bos et al. 2012; Crockford et al. 2014; Heinrichs et al. 2009; Insel 2010; Mehta \& Josephs 2012; van Anders et al. 2011). To what extent these behaviors play a role in teachers or pupils may depend upon the teaching type. Therefore, we propose that Kline's teaching types can be mapped to hormonal variations in teachers and learners via social and cognitive building blocks (Fig. 1). This approach parallels existing frameworks for the study of cooperation (Soares et al. 2010), and according to Kline, teaching is a cooperative behavior.

Kline proposes to conduct comparative research with emphasis on socio-environmental niches in which teaching and specific teaching types evolve. In cooperatively breeding New World monkeys, after the birth of an infant, fathers experience changes in vasopressin, oxytocin, and testosterone (Kozorovitskiy et al. 2006) and siblings show increases in oxytocin (Ragen \& Bales 2012), suggesting physiological adaptations to infants and juveniles (the individuals who are usually taught). Rearing conditions influence later oxytocin balance and social behavior (Fries et al. 2005; Winslow et al. 2003), and altruistic behaviors, sibling

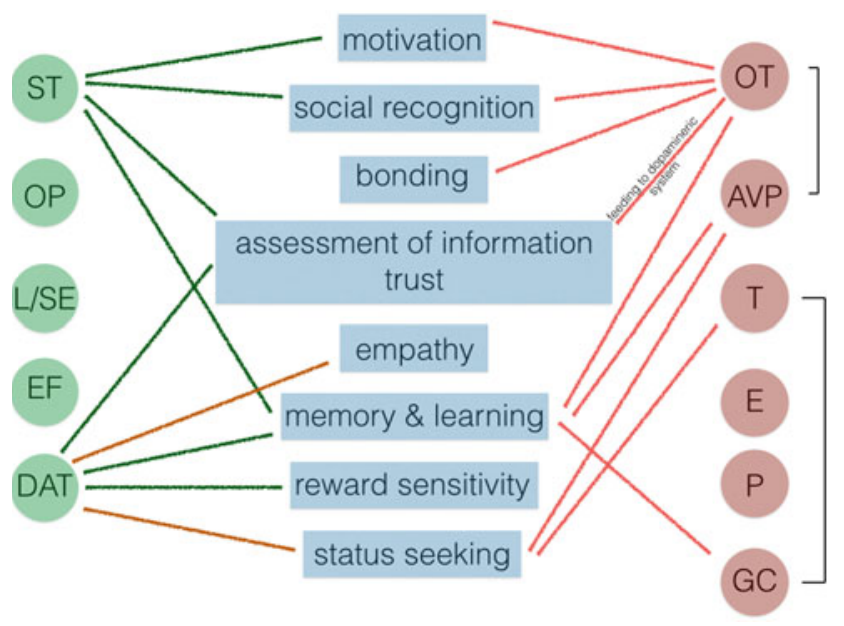

Figure 1. (Ravignani \& Sonnweber). Exemplary mapping of teaching types to hormonal measures via behaviors. The "building blocks" of teaching (in the middle with blue background) are mediated by and fed back to different hormones (right with pink background), such as oxytocin (OT), vasopressin (AVP), testosterone (T), estrogens (E), progesterone (P) or glucocorticoides (GC) (actual although incomplete results on behavior-hormone interactions are indicated with red lines). Hormonal measures allow the investigation of motivational and emotional changes in teaching contexts and can be linked to cognitive processes and behavioral modifications associated with teaching. Kline distinguishes five teaching types based on the adaptive problem they solve: (i) teaching by social tolerance (ST), (ii) opportunity provisioning (OP), (iii) local or stimulus enhancement (L/SE), (iv) evaluative feedback (EF), and (v) direct active teaching (DAT). A precise mapping between building blocks and different teaching types needs to be investigated: Predictions on possible connections are indicated on the left (brown lines for teachers and green lines for pupils). Mapping teaching types to hormones and behaviors may help us understand basic processes and mechanisms of teaching across and within species.

relationships, or decision making are genetically associated with different vasopressin-receptor types (Israel et al. 2008; Knafo et al. 2008). Parental investment and siblings' infant care predict changes in vasopressin and oxytocin in cooperatively breeding monkeys (Ragen \& Bales 2012). Hence, developmental and epigenetic forces might contribute to the evolution of teaching behavior (Bjorklund 2006; Soares et al. 2010). Future comparative data will elucidate the epigenetics of teaching.

While hormones elucidate internal states, Kline's focus is on external, observable behaviors. She claims that the only example of direct active teaching in nonhuman animals comes from anecdotes of chimpanzees learning to crack nuts (Boesch 1991). Building on recent work on synchrony and motor mimicking in chimpanzee dyads (Fuhrmann et al. 2014), we propose additional tools to measure teaching and learning over time across species and behaviors.

A chimpanzee performing quasi-periodic movements to crack nuts can be tracked over time, for example, via video coding (Fuhrmann et al. 2014) or movement sensors (Nagasaka et al. 2013; Ravignani et al. 2013). This produces, for each individual, evenly spaced samples (time series) of rhythmic, learnable behaviors. Behaviors can be movements, fundamental frequency of vocalizations, or any other possible recordable semi-repetitive behavior within short time scales (few seconds). Kline stresses the importance of comparing behaviors in teaching and nonteaching contexts, and argues that finding differences in rates of behaviors between baseline and teaching contexts suffices to 
conclusively demonstrate teaching. Time series of teachers and pupils can be plotted together and statistically related to test hypotheses on teaching types.

Autocorrelation (correlation of a series with itself at different time lags) can be employed to investigate practice and self-consistency in learning movement patterns. Increased learning can be shown via an increase in between-trial autocorrelation (i.e., increased predictability of the pupil's next step once the action is almost completely learned).

Faithfulness of action copying and individual learning performance can be investigated using cross-correlations: the higher the correlation between teacher and pupil, the more accurate the learning. A cross-correlogram provides a measure in the delay of copying: A high cross-correlation (near zero lag) provides evidence for simultaneity of actions (high cross-correlation at a short lag is predicted in stimulus/local enhancement). Alternative methods, originally developed to infer similarity between geometrical curves, can measure resemblance between taught/learnt behaviors, such as Fréchet distance (Alt \& Godau 1995), procrustes analysis (Gower 1975), and dynamic time warping (Verhoef et al. 2014).

Granger-causality (Granger 1969; Seth 2010) enables investigation of directionality of information transmission in the teaching process; a teacher's time series causes a pupil's time series (sensu Granger-causality) if past teacher's data significantly improve the prediction of future pupil's data (when compared to forecasts based on past pupil's data alone). Granger-causality can be used to show that teacher-pupil synchrony is unilaterally driven by one of the two (Fuhrmann et al. 2014). Alternatively, two time series Granger-causing one another constitute evidence for bilateral information transmission: not only does the pupil's series depend upon the teacher's series, but the teacher's behavior will also be triggered by a pupil's (imperfect) behavior (as needed in evaluative feedback). An alternative for measuring the amount and directionality of information transmission is partial directed coherence (Baccalá \& Sameshima 2001; Ghazanfar et al. 2012).

The proposed quantitative tools can serve to analyze behaviors in teaching contexts. Hormonal measures allow for conclusions about motivational and emotional states or reward mechanisms. Controlled correlation studies measuring relevant hormones (i.e., via saliva, urine, or feces) or experimental administration studies can help shed light on basal processes involved in teaching and social learning. The tools we suggest here will hopefully contribute to a more empirical and quantitative approach to teaching, transcending verbal descriptions alone.

\section{ACKNOWLEDGEMENTS}

This work was supported by Austrian Academy of Sciences grant "Learning from a Friend" to W. Tecumseh Fitch and Ruth Sonnweber, and European Research Council Advanced Grant 230604 SOMACCA to W. Tecumseh Fitch (supporting Andrea Ravignani and Ruth Sonnweber). We thank Gesche Westphal-Fitch for comments and Barbara Finlay for extremely helpful advice.

\section{NOTE}

1. Andrea Ravignani and Ruth Sonnweber contributed equally to this commentary as joint first authors.

\section{The benefits of an evolutionary framework for the investigation of teaching behaviour: Emphasis should be taken off humans as a benchmark}

\author{
doi:10.1017/S0140525X14000582, e59
}

Amanda R. Ridley ${ }^{\mathrm{a}, \mathrm{b}}$ and Benjamin J. Ashton ${ }^{\mathrm{a}}$
${ }^{\mathrm{a} C e n t r e}$ of Evolutionary Biology, School of Animal Biology, University of
Western Australia, Crawley, Perth, WA 6009, Australia; ${ }^{\mathrm{b}}$ Percy FitzPatrick
Institute of African Ornithology, University of Cape Town, Cape Town, WC 7701, South Africa.

Amanda.ridley@uwa.edu.au www.babbler-research.com

http://www.ceb.uwa.edu.au/research/profiles?profile/1/id/4130

Abstract: We agree with Kline that a lack of unification is preventing progress in understanding the occurrence of teaching behaviour and the selective pressures influencing its presence. However, we feel that the proposed framework, which incorporates mentalistic and cultural approaches, continues to overlook cases of teaching in nonhuman animals. We advocate the comparative functionalist framework to identify the proximate causes of teaching behaviour in both humans and other animals.

Teaching is a behaviour, or array of behaviours, that has provoked considerable debate (see Csibra 2007; Hoppitt et al. 2008; Premack 2007; Thornton \& Raihani 2008). Much of this debate comes from the belief that teaching is a behaviour that requires uniquely human cognitive abilities, such as theory of mind, and therefore occurs only in humans (Premack 2007). A number of supporters of the mentalistic and culture-based approaches to teaching argue that there is remarkably little evidence for teaching in nonhuman animals, and supporters of the functionalist approach agree (Byrne et al. 2013; Thornton \& Raihani 2008; 2010). However, the way that teaching is defined is likely to be a primary cause for the relative absence of it in nonhuman animals (Thornton \& Raihani 2010). Indeed, a recent surge in research claiming to show teaching in nonhuman animals has benefited strongly from the functionalist definitions proposed by Caro and Hauser (1992) and updated by Hoppitt et al. (2008). By providing a definable criteria for what constitutes teaching, within a quantifiable and comparative framework, teaching has now been discovered in a number of animals (for recent examples, see Franks \& Richardson 2006; Kleindorfer et al. 2014; Raihani \& Ridley 2008; Thornton \& McAuliffe 2006; and see Maestripieri et al. [2002] and others reviewed in Thornton \& Raihani [2008] for anecdotal examples of teaching in animals that have not yet been experimentally proven).

Teaching, if we are to follow the functionalist definition, may therefore not be as rare as originally supposed. Indeed, Byrne et al. (2013) argue that "the old idea that cultural learning through teaching is how we do things, while trial-and-error fumbling is how animals do them has now been thoroughly discredited" (p. 51 [emphasis in original]).

Even from the perspective of human-based studies it has become increasingly acknowledged that the mentalistic and culture-based definitions of teaching are overly restrictive. For example, recent research into the occurrence of teaching behaviour in humans has used nonhuman examples to explain the different types of teaching that exist (Dean 2011; Niedermeyer 2014). The famous philosopher John Dewey wanted to ground our understanding of teaching in evolutionary theory, and hence came up with a definition of the two types of teaching - which is very similar to that of Thornton and Raihani (2008). However, Dewey failed to make the explicit link between the evolutionary bases of teaching behavior and human education (Niedermeyer 2014). Thus, Kline's article provides welcome recognition of the need for a unification of the definition of teaching in her call for a new, evolutionary framework for teaching.

Previously, it has been suggested that teaching must be defined in the absence of environmental and genetic influences (reviewed in Dean 2011). However, increasing opposition to this idea identifies that environmental factors are likely to influence behavioural patterns in all animals, and to exclude them would result in false negatives (Laland \& Janik 2006). Therefore, while we agree with Kline that a new framework for the definition and measurement of teaching behaviour is required, we disagree with the following statement:

[A]ny framework for understanding the evolution of teaching should be tested against the human case. (target article, sect. 2.4, para. 6) 